\title{
The Impact of Strontium Ranelate on Implant Osseointegration of Osteoporosis in Vivo Experiment
}

\author{
Maiquan Wang ${ }^{1}$, Qingqing Liu ${ }^{2}$, Chao Yang ${ }^{2}$ and Yuanyuan Zhang ${ }^{2^{*}}$ \\ ${ }^{1}$ People's Hospital of Zhengzhou University, Zhengzhou Henan 450003, China \\ ${ }^{2}$ Department of Oral Medicine, College of Stomatology, Zhengzhou University, Zhengzhou, Henan 450052, China
}

"Corresponding author: Dr. Yuanyuan Zhang, Department of Oral Medicine, Zhengzhou University of School of Stomatology, 40 Zhongyuan Road, Zhengzhou, 450052, China; E-mail: zhyy143@126.com

Rec date: Apr 18, 2016; Acc date: May 10, 2016; Pub date: May 18, 2016

Copyright: (c) 2016 Wang M, et al. This is an open-access article distributed under the terms of the Creative Commons Attribution License, which permits unrestricted use, distribution, and reproduction in any medium, provided the original author and source are credited.

\begin{abstract}
Objective: To investigate the impact of strontium ranelate on implant osseointergration of osteoporotic rats in vivo experiment.

Method: Thirty-six female rats were randomly divided into three groups: ovariectomization group $(A=12)$, strontium ranelate low-dose treatment group $(B=12)$ and strontium ranelate high-dose treatment group $(C=12)$. After the success of the osteoporosis model building, HA coated titanium implants were inserted into proximal metaphyses of tibiae, meanwhile, the rats of group $B$ and $C$ received oral medication of strontium ranelate (B: 450 $\mathrm{mg} / \mathrm{kg} . \mathrm{d} \mathrm{C}$ : $900 \mathrm{mg} / \mathrm{kg} . \mathrm{d}), 12$ weeks after surgery, animals were killed and decalcified sections were prepared and examined histologically and histomorphometrically, as well as micro-CT examination.
\end{abstract}

Results: 12 weeks after implantation surgery, Bone Mineral Density (BMD), Implant Bone Contact Rate (IBCR) and New Bone Volume (NBV) in group B and C were significantly higher compared with group $A(p<0.01)$.

Conclusion: Strontium ranelate may resist the negative influence of osteoporosis and promote osseointergration of implant.

Keywords: Strontium ranelate; Osteoporosis; Implant; Osseointeration; Bone mineral density; Implant bone contact rate; New bone volume

\section{Introduction}

Dental implant restoration is one of the most important methods for tooth loss therapy. Osteoporosis (OP) has been regarded as an important risk factor for implant failure [1,2] and clinical studies have demonstrated that OP could reduce the osseointegration of implant, it is necessary to enhance the bone metabolism and inhibit excessive bone resorption on the surface of implant [3].

Strontium ranelate (SR), a new active drug shown to reduce OP, can stimulate osteoblast proliferation and inhibit osteoclast resorption, which allowing promoting bone formation while decreasing bone resorption [4-7]. SR has a good application prospect and was designed to contribute to the dental implant in postmenopausal women with OP. In recent years there was a growing number of studies investigated the effect of SR for treating OP, and the result showed that it could increase the bone weight of vertebra, humerus, and femur of animals.

However, rarely studies reported that SR was used for dental implant and increasing bone volume around the implant area in patients suffered from OP. The purpose of our study was to investigate the effect of strontium ranelate on implant osseointergration in $\mathrm{OP}$ rats.

\section{Material and Methods}

\section{Materials}

Total amount of 36 female Sprague Dawley (SD) rats (3 months old) with an average weight of $250 \mathrm{~g}$ were purchased from Laboratory Animal Center of Henan Province. Hydroxyapatite (HA) coated titanium implants were provided by Biomaterials Testing Center of Sichuan University. SR was purchased from Servier Company (France) and the Dual-energy X-ray absorptiometry (DEXA) was made in USA.

\section{OP induction in SD rats}

Animals were induced through bilateral ovarian wedge resection [8] and randomly divided into three groups, the OP group (group A), the low-dose SR treatment group (group B) and the high-dose SR treatment group (Group C). Animals were given dexamethasone $(0.5$ $\mathrm{mg} / \mathrm{kg}$ ) subcutaneous injections after surgery twice a week for 12 weeks.

\section{Dental implantation and SR administered}

12 weeks after OP induced, HA coated titanium implants were implanted into the right tibiae near metaphysical. For all the SD rats, the procedures were as follows: incised the skin and soft tissue of the right tibiae near metaphysical to expose the bone, prepared planting holes with a $2 \mathrm{~mm}$-diameter dental drill, screwed the implants penetrating into the cortical bone and layered suture incisions. Conventional farming, penicillin $\left(40 \times 10^{4} \mathrm{U} / \mathrm{Kg}\right)$ was intramuscular 
administered 1 day before and everyday post-surgery for 3 days, respectively. Rats in group B (450 mg/kg a day) and C $(900 \mathrm{mg} / \mathrm{kg}$ a day) were given SR orally.

\section{Histological observation and bone histomorphometric analysis}

12 weeks after implantation, animals were sacrificed. And the tibial implants region (20 $\mathrm{mm}$ in length) were excised and embedded in resins. And hard tissue slices at the thickness of 4 um with undecalcification were obtained by using a slice microtome, and all the slices were stained by using methylene blue. Microstructures of the implant-bone interface (trabecular bone around implants) were observed with a micro-CT. Dual energy X-ray absorptiometry was used for measuring the bone mineral density (BMD).

The digital medical analysis system was adopted for analysis of the following parameters in the implant region:

- Implant Bone Contact Rate (IBCR \%), which was the proportion of direct contact area of the implant and newly formed bone account for the total area of the implant.

- New Bone Volume (NBV \%), which was the proportion of area of the new bone formed within $1 \mathrm{~mm}$ around the thread bottom of the implant account for the total area of this region.

\section{Statistical analysis}

Statistical analyses were performed using the statistics package SPSS16.0. One-way ANOVA was used to compare the BMD, IBCR and NBV among the 3 groups. The level of significance was set at $\mathrm{p}<0.01$.

\section{Results}

\section{The measurement of BMD, IBCR, NBV}

After 12 weeks, BMD, IBCR, NBV in group B and group C were significantly higher than group $A(p<0.01),{ }^{*} p<0.01$ and there was a significant difference compared with the control group. But no statistically significant difference between group B and C given in the Table 1.

\begin{tabular}{|l|l|l|l|}
\hline & BMD & IBCR (\%) & NBV (\%) \\
\hline Group A & $0.1958 \pm 0.0173$ & $43.25 \pm 4.01$ & $42.76 \pm 5.66$ \\
\hline Group B & $0.2627 \pm 0.0185^{*}$ & $67.25 \pm 5.50^{*}$ & $73.43 \pm 6.06^{*}$ \\
\hline Group C & $0.2855 \pm 0.0179^{*}$ & $70.05 \pm 6.50^{*}$ & $75.78 \pm 6.23^{*}$ \\
\hline * $p<0.01$ & & \\
\hline
\end{tabular}

Table 1: comparison of each index after different dose SR was administered $(\mathrm{X} \pm \mathrm{S})$

\section{Discussions}

$\mathrm{OP}$ is a systemic bone disease characterized by low bone mass, damage of bone microstructure, increased bone fragility and being more vulnerable to fracture. It can be happened in different gender and age groups, especially in aged men and postmenopausal women [9]. OP which happened in postmenopausal women is a bone abnormal metabolism disease that the bone resorption outweights bone formation accompanies estrogen deficiency. Pathological anatomy showed thining cortical, more porous and interruptions trabecular [10]. When OP happened, the IBCR and the relative bone mass were decreased significantly in the OP area. In our study, the OP model was built in rats through bilateral ovarian wedge resection combined with dexamethasone injection.

SR, under the brand name Protelos, was developed by Servier company (France). It was a drug for OP treatment with a dual mechanism targeting decreasing bone resorption and increasing bone formation. Currently, numerous animal experiments have demonstrated that Protelos could accelerate the new bone formation and slow down the old bone resorption. Study showed that Protelos could both decrease the bone dropping in ovariectomized rats and enhance the bone density in normal animals [11]. SR has dual pharmacological effects. Regarding the proliferation of the osteoblast, the SR can increase the collagen and non-collagen protein synthesis, at the same time; it can promote the osteoblast-mediated bone formation by promoting the proliferation of the preosteoblast. Simultaneously, it can play a role in inhibiting the differentiation of the pre-osteoclast in a concentration-dependent manner, consequently inhibiting the osteoclast-mediated bone resorption. Some studies indicate that SR treatment may increase the risk of cardiac disease, but SR is not limited and it is still retained as a therapeutic option for osteoporosis. When used appropriately, it should therefore remain part of therapeutic armamentarium for osteoporosis [12].

This paper investigated the effect of different doses of SR on the Osseo integration of titanium implant in OP animals by the methods of histologic examination, micro-CT, BMD, etc. Results showed that bone trabeculae in the implant region in the low-dose group were significantly larger than group A, but less in quantity and thinner in thickness. It can be observed that the bone formation area was larger in the high-dose group. BMD, IBCR, NBV of the group B and group C significantly increased $(\mathrm{P}<0.01)$ and were higher than group $A$ after 12 weeks $(\mathrm{P}<0.01)$. The utility of SR could minimize the negative effects of $\mathrm{OP}$, promote the healing of surrounding bone implant body and increase the implant-bone contact ratio. Figure 1 (results of histologic examination) and Figure 2 (results of micro-CT) showed that with the increasing of SR dose, the bone trabecular formation and bone mass increased. Compared with the control group, the intake of SR was more, the bone formation was more. Continuous and large area of bone formation could be observed in group $\mathrm{B}$ and group $\mathrm{C}$, and were more than group A significantly $(\mathrm{P}<0.01)$. However, there was no significant difference between group $\mathrm{B}$ and group $\mathrm{C}$.

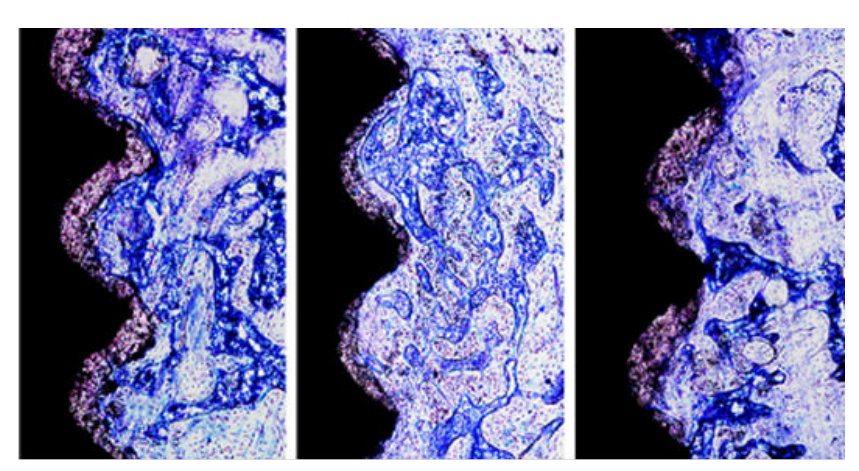

Figure 1: Implant-bone interface morphology 12 weeks after surgery $(100 \times)$ 
Citation: Wang M, Liu Q, Yang C, Zhang Y (2016) The Impact of Strontium Ranelate on Implant Osseointegration of Osteoporosis in Vivo Experiment. Dentistry 6: 376. doi:10.4172/2161-1122.1000376

Page 3 of 3

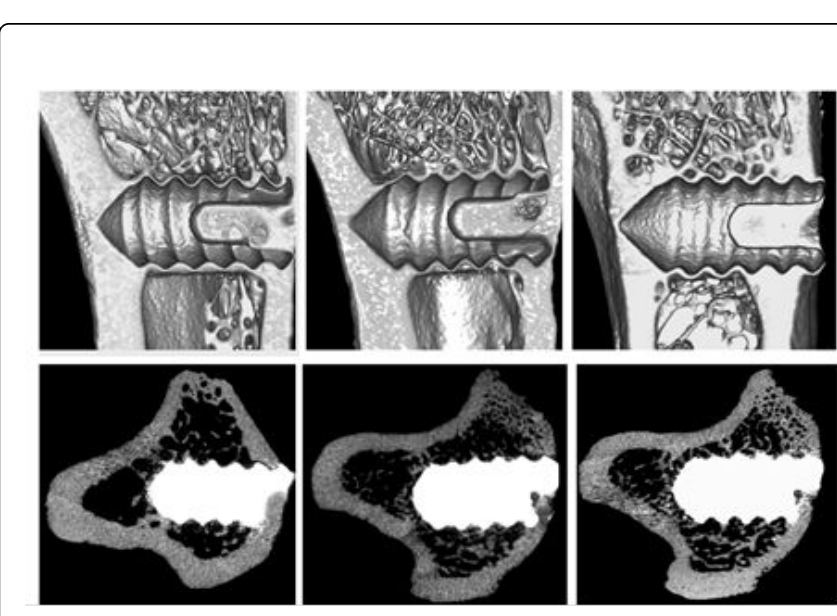

Figure 2: Implant bone trabecular microstructure 12 weeks after surgery

Ammann et al. [13] found that different dose Protelos (450,900 mg/ kg.d) could enhance bone strength at different levels, and increase bone mass of vertebra and femur in rats. Protelos could augment the volume of the trabecular and cortical bone volume, and increase the total thickness and amoun of trabecular [14]. It could also improve the basic serum Insulin-like Growth Factor (IGF) and plasma Alkaline Phosphatase (ALP) level. The SR dose adopted in our study was determined according to the previous studies and literatures review. This study has shown that the bone formation after SR intake differ significantly from the OP group. The results indicated that the different effects of different oral dose in the study were obvious. However, whether the dose is appropriate for dental implant or not need further study to explore. To obtain an ideal bone mass of the implant region, the optimum oral dose and frequency of SR should be further studied.

\section{Acknowledgement}

This experiment on female rats was performed after taking permission from Animal Ethics Committees (AECs) and it was monitored, supervised by the head of Department of Oral Medicine, Zhengzhou University of School of Stomatology, China.

\section{References}

1. Hwang D, Wang HL (2007) Medical contraindications to implant therapy: Part II: Relative contraindications. Implant Dent 16: 13-23.

2. Bornstein MM, Cionca N, Mombelli A (2009) Systemic conditions and treatments as risks for implant therapy. Int J Oral Maxillofac Implants 24 Suppl: 12-27.

3. Li Y, Gao Y (2010) Additive effects of estrogen replacement therapy and bisphosphonates on osseointegration of hydroxyapatite-coated itanium screws in ovariectomized rats. Oral and maxillofacial implants 109: 700-705.

4. Jiang $\mathrm{H}$ (2006) Rainie acid strontium pharmacodynamic and pharmacokinetic research progress. Chinese journal of hospital pharmacy 26: 197-199.

5. Bonnelye E, Chabadel A, Saltel F, Jurdic P (2008) Dual effect of strontium ranelate: stimulation of osteoblast differentiation and inhibition of osteoclast formation and resorption in vitro. Bone 42: 129-138.

6. Bain SD, Jerome C, Shen V, Dupin-Roger I, Ammann P (2009) Strontium ranelate improves bone strength in ovariectomized rat by positively influencing bone resistance determinants. Osteoporos Int 20: 1417-1428.

7. Arlot ME, Jiang Y, Genant HK, Zhao J, Burt-Pichat B, et al. (2008) Histomorphometric and microCT analysis of bone biopsies from postmenopausal osteoporotic women treated with strontium ranelate. J Bone Miner Res 23: 215-222.

8. Kalu DN (1991) The ovariectomized rat model of postmenopausal bone loss. Bone Miner 15: 175-191.

9. Huiping M, Zhengping J, Xin G (2004) Research status of prevention and cure of osteoporosis drugs. china pharmacist 7: 816-818.

10. Kasugai S (2006) Dental implant treatment to osteoporosis patients. Clin Calcium 16: 348- 353.

11. Komrakova M, Weidemann A, Dullin C, Ebert J, Tezval M, et al. (2015) The Impact of Strontium Ranelate on Metaphyseal Bone Healing in Ovariectomized Rats. Calcif Tissue Int 97: 391-401.

12. Compston J (2014) Strontium ranelate lives to fight another day. Maturitas 78: 75-76.

13. Ammann P, Shen V, Robin B, Mauras Y, Bonjour JP, et al. (2004) Strontium ranelate improves bone resistance by increasing bone mass and improving architecture in intact female rats. J Bone Miner Res 19: 2012-2020.

14. Blake GM, Fogelman I (2006) Strontium ranelate: a novel treatment for postmenopausal osteoporosis: a review of safety and efficacy. Clin Interv Aging 1: 367-375. 